\title{
The Effects of Trunk Exercise on Mobility, Balance and Trunk Control of Stroke Patients
}

\author{
Seung-Heon An - Dae-Sung Park ${ }^{1 \dagger}$
}

Dept. of Physical Therapy, National Rehabilitation Hospital

${ }^{1}$ Dept. of Physical Therapy, Konyang University

Received: September 16, 2016 / Revised: September 26, 2016 / Accepted: October 13, 2016

(c) 2017 J Korean Soc Phys Med

\section{| Abstract |}

PURPOSE: The initiation of the trunk muscles in stroke patients is delayed because the muscles involved in reach arm are activated earlier than the trunk muscles. The objective of this study was to examine the effects of mobility, balance, and trunk control ability through selective trunk exercise (STE) in patients with chronic stroke.

METHODS: A randomized pre-test and post-test control group design was initially used, with subjects randomly assigned to the STE group $(n=15)$ and a control group $(n=14)$. All groups underwent physical therapy based on the neuro-developmental therapy (NDT) for 30 minutes a day, five times per week for four weeks. Additionally, the STE group did the trunk exercise for 30 minutes a day, three times per week for four weeks. The timed up and go test (TUG), Berg balance scale (BBS), and trunk impairment scale (TIS) were used for assessment.

RESULTS: The scores of the TUG, BBS, dynamic sitting balance subscale, and coordination subscale of TIS improved significantly in both groups but the improvement was more

$\dagger$ Corresponding Author : daeric@konyang.ac.kr This is an Open Access article distributed under the terms of the Creative Commons Attribution Non-Commercial License (http://creativecommons.org/licenses/by-nc/3.0) which permits unrestricted non-commercial use, distribution, and reproduction in any medium, provided the original work is properly cited. pronounced in the STE group $(\mathrm{p}<.05)$. This study showed a large effect on the scores of the TIS coordination subscale $(\mathrm{d}=.93)(\mathrm{p}<.05)$, TIS dynamic balance subscale $(\mathrm{d}=.81)$ $(\mathrm{p}<.05)$, TUG $(\mathrm{d}=.75)(\mathrm{p}<.05)$, and BBS $(\mathrm{d}=.73)(\mathrm{p}<.05)$.

CONCLUSION: The combined STE and NDT program showed improvements in measures of mobility, balance, and trunk control in chronic stroke patients. These results suggest that STE should be considered to be included in the treatment program for patients with chronic stroke.

Key Words: Berg balance scale, Stroke, Timed up and go, Trunk exercise, Trunk impairment scale

\section{Introduction}

Trunk control requires good sensomobility in order to maintain and recover the balance of body (Karatas et al., 2004; Ryerson et al., 2008). The trunk muscles are involved in selective movements to control the center of gravity as the base of support during standing, weight shifting, and maintaining static and dynamic postures (Verheyden et al., 2004). In general, the trunk muscles of a healthy person engage in controlling initial posture and are activated before the movement of the upper and lower limbs. However, when it comes to stroke patients, it is known that the initiation of the trunk muscles is delayed, because 
the muscles involved in reach arm are activated earlier than the trunk muscles (Dickstein et al., 2000). Moreover, a comparison test using isokinetic dynamometer for the powers of trunk flexors, extensors, and both rotation muscles showed that stroke patients had statistically significant weaker trunk muscles than healthy people (Bohannon, 1992). In a proprioceptive position sense test of the trunk, stroke subjects showed an inability to re-position from the starting posture, compared to healthy people in the three planes: sagittal, frontal, and horizontal (Ryerson et al., 2008; Han and Shin, 2013).

By contrast to hemiplegia associated with the opposite side of brain lesions in post-stroke patients, trunk muscles have a multifaceted disorder (Bohannon, 1992; Dickstein et al., 1999; Fujiwara et al., 2001; Tsuji et al., 2003). In practice using the trunk impairment scale (TIS), amongst sub-acute stroke patients with trunk movement impairment are early predictors of the duration of hospital stay and performance of daily living activities (Duarte et al., 2002; Verheyden et al., 2006). Also, trunk movement impairment associated with balance $(51 \%)$, walking speed $(27 \%)$ and functional mobility (44\%) (Verheyden et al., 2006). The trunk movement impairment and muscle weakness in stroke patients can have a negative impact on the control of position and independent activities of daily living. Therefore, recovering the ability of trunk control should be preceded by the training to restore other functions (Song and Kim, 2010; Jang and Kim, 2016).

The disorders of stroke patients, such as the weakness of trunk muscles, inappropriate motor-unit recruitment, the impairment of neurologic control and proprioceptive sense, excessively use abnormal compensatory strategies owing to disuse syndrome (Han and Shin, 2013). Exercise is focused on the restoration of upper and lower limbs, balance, and walking, rather than trunk exercise.

The previous study demonstrated that the effect of the trunk lateral flexion exercise in sitting among sub-acute stroke patients (Verheyden et al., 2009) and the improvement in symmetric sitting posture among acute stroke patients through recognition training of trunk location (Mudie et al., 2002). The previous study (Karthikbabu et al., 2011b) about the effectiveness of trunk exercise using a plinth in chronic stroke patients, the results cannot be generalized as it did not have a control group. Therefore, this study aims to explore how additional trunk muscle training can be effective for mobility, balance, and trunk control of chronic stroke patients.

\section{Methods}

The participants of the study were recruited from "M" hospital. The criteria for subjects included patients with chronic stroke after six months of diagnosis, having a higher score than 24 in mini mental state examination-korean version (MMSE-K), being able to walk 10 meters independently, and scoring less than 21 on the trunk impairment scale (TIS). Therefore, patients with scores higher than 21 on the TIS were excluded from this study as it indicates that they can perform everyday activities independently (Verheyden et al., 2005).

This research was conducted between May 2014 and November 2014. The twenty-eight patients with chronic stroke participated for the study were observer-blinded, randomized through the block randomization method, block size of $2 \times 2$. The method of allocation was concealed in sequentially numbered, sealed envelopes. An independent observer who does not involved in interventions and the outcome measures performed the randomization. Allocated into two groups; 19 participants were in the selective trunk exercise (STE) group and 19 in the control group. All groups received conventional physical therapy based on the neuro-development therapy 30 minutes per day, five times per week for four weeks. It applied that stretching 
exercise, strengthening of lower extremity muscle, progressive gait training, balance control, weight shifting, bearing.

The exercise programs of STE group were applied the protocols which suggested of the previous studies (Karthikbabu et al., 2011a; 2011b). The exercise program consisted of four supine exercises and seven sitting exercises. The four supine exercises were lifting the pelvis with crook-lying, unilateral pelvic bridge, upper trunk flexion rotation, and lower trunk flexion rotation. The seven sitting exercises were as follows: selective flexion extension of the lower trunk, upper trunk lateral flexion, lower trunk lateral flexion, upper trunk rotation, lower trunk rotation, forward reach, and lateral reach. The forward and lateral reach were performed at shoulder height.

The frequency of exercise was in accordance to the patients' physical performance capabilities. The exercise intensity was controlled by the reduction of the base support, an increase in lever arm to intensify the exercise load, and the changes in maintaining time. In addition, when the patients were training, we supported them to minimize compensatory movements and to move the trunk of patient, diminishing observation and verbal advice. We conducted warm-up and cool down sessions for two minutes. There were 1-2 minute breaks during each exercise and 30 minutes per a day, three times per week for four weeks.

Subjects were required to stand up from a chair with arm rests, walk $3 \mathrm{~m}$, turn around, return to the chair, and sit down. The time taken to complete this task was measured in seconds with a stopwatch (Podsiadlo and Richardson, 1991). The test-retest reliability of TUG test in subjects with chronic stroke showed a high degree intra class correlation ( $\mathrm{ICC}=.96)$ (Flansbjer et al., 2005).

The Berg balance scale (BBS) consists of 14 different tasks. The tasks test the subject's ability to maintain positions or movements of increasing difficulty as well as the ability to change positions. Each item is scored on a scale of 0 to 4 , for a maximum of 56 points. A change in the score of 5 points has been shown to be clinically significant when used on a pre-test and post-test basis. The test-retest reliability in subjects with chronic stroke showed a high degree (ICC=.95) (Mao et al., 2002).

The assessment for trunk impairment such as paresis was carried out on the TIS. The TIS consists of three subscales: static sitting balance, dynamic sitting balance and coordination, containing 3,10, and 4 items. The TIS score ranges from a minimum of 0 to a maximum of 23 , with a higher score indicating a better trunk performance. The static subscale (a score of 7) investigates the ability of the subject to maintain a sitting position with the feet supported, while the legs are passively and actively crossed. The dynamic subscale (a score of 10) contains items on lateral flexion of the trunk and unilateral lifting of the hip. The coordination subscale (6 score) of the trunk contains selective rotation of the upper and lower part of the trunk. Test-retest reliability was ICC $=.94 \sim .97$ (Verheyden et al., 2005).

We compared general characteristics between the STE group and the control group using the chi-square test. The independent t-test was conducted to analysis the change of different between the two groups. The paired t-test was used to compare the difference between pre- and post-intervention scores within the group. The level of significance was set at $\mathrm{p}<.05$. The analysis was performed using the SPSS version 18.0 (SPSS Inc., USA). The effect size was computed using the formula $d=d / s(d)$ where $d$ is the mean difference scores, and s (d) is the standard deviation of the difference scores. Effect size index was then defined using Cohen's classification of effect size index (d), where small $d=.20$, medium $d=.50$ and large $d=.80$. Hopkins (2000) suggests that a sample size of at least 30 individuals should be considered in reliability studies, in this case the sample size was 31 using $\mathrm{G}^{*}$ power software ver 3.1.9.2 (Kiel University, Germany) on effect size .85 , alpha $=.05$, power $=.95$. 


\section{Results}

Twenty-seven patients completed the study. One patient in control group was lost to follow up at three weeks. The data collected in this study was from 15 participants in the intervention group and 14 from the control group. Table 1 shows the participants demographic characteristics. When comparing the mean pre-intervention and postintervention differences using an independent sample $t$ test, TUG of the experimental group significantly decreased more after intervention than the control group $(p<.05)$. The BBS score significantly increased more in the experimental group than the control group $(p<.05)$. The effect size Cohen $d$ index for TUG (.75) $(p<.05)$ and BBS $(.73)(p<.05)$ were a large value (Table 2).
In the pre-post-tests within the groups, TIS-static did not have a significant difference, but TIS-total, TIS-dynamic and TIS-coordination increased $1.56(p<.05), .98(p<.05)$, and $.59(p<.05)$ more in each than the control group. The effect size Cohen $d$ index for coordination $(.93)(p<.05)$ and static $(.81)(p<.05)$ were reached a power value of .80 . The effect size index (Cohen $d$ ) for TIS-coordination $(.98)(p<.05)$ and TIS-dynamic $(.81)(p<.05)$ were large compared to TIS-static $(.02)(p<.05)$ (table 3$)$.

\section{Discussion}

There is an impairment in the ability to control the trunk for post-stroke patients due to excessive use and learning

Table 1. Demographic characteristics of participants

\begin{tabular}{lcccc}
\hline & STE group $(n=15)$ & Control $(n=14)$ & $X^{2} / t$ & $p$ \\
\hline Age (years) & $59.73 \pm 8.94$ & $57.07 \pm 17.17$ & .529 & .601 \\
Gender (male/female) ${ }^{\dagger}$ & $7 / 8$ & $8 / 6$ & .318 & .715 \\
Hemiplegic side (left/right) ${ }^{\dagger}$ & $8 / 7$ & $9 / 5$ & .358 & .710 \\
Stroke lesion (ischemic/hemorrhage) ${ }^{\dagger}$ & $9 / 6$ & $8 / 6$ & .024 & 1.00 \\
Duration (month) & $9.07 \pm 3.47$ & $8.93 \pm 2.30$ & .125 & .901 \\
\hline
\end{tabular}

${ }^{\dagger}$ Pearson chi-square

STE group: selective trunk exercise group

Table 2. Comparison of timed up and go test, Berg balance scale in pre-post test

$(n=29)$

\begin{tabular}{|c|c|c|c|c|c|c|}
\hline & STE group $(n=15)$ & Control $(n=14)$ & $t$ & $p$ & Cohen $d$ & Power \\
\hline \multicolumn{7}{|c|}{ TUG (seconds) } \\
\hline pre & $18.02 \pm 4.77$ & $16.37 \pm 6.89$ & .753 & .458 & & \\
\hline post & $13.91 \pm 3.50$ & $14.37 \pm 4.29$ & & & & \\
\hline different & $-4.11 \pm 2.24$ & $-2.01 \pm 3.05$ & 2.124 & $.043^{*}$ & .75 & .53 \\
\hline $\mathrm{t}$ & $7.088^{*}$ & $2.464^{*}$ & & & & \\
\hline \multicolumn{7}{|l|}{ BBS (scores) } \\
\hline pre & $41.20 \pm 4.14$ & $42.07 \pm 3.95$ & .579 & .568 & & \\
\hline post & $44.13 \pm 2.72$ & $43.43 \pm 3.11$ & & & & \\
\hline different & $3.07 \pm 2.66$ & $1.36 \pm 1.60$ & 2.079 & $.047^{*}$ & .73 & .52 \\
\hline $\mathrm{t}$ & $4.363^{*}$ & $3.177^{*}$ & & & & \\
\hline
\end{tabular}

$* p<.05$,

STE group: selective trunk exercise group, TUG: timed up and go test, BBS: Berg balance scale 
The Effects of Trunk Exercise on Mobility, Balance and Trunk Control of Stroke Patients | 29

Table 3. The compare of trunk impairment scale in pre-post test

$(n=29)$

\begin{tabular}{|c|c|c|c|c|c|c|}
\hline Item (score) & STE group $(n=15)$ & Control $(n=14)$ & $t$ & $p$ & Cohen $d$ & Power \\
\hline \multicolumn{7}{|l|}{ TIS-static (7) } \\
\hline pre & $6.27 \pm .59$ & $6.29 \pm .47$ & .095 & .925 & & \\
\hline post & $6.45 \pm .52$ & $6.50 \pm .52$ & & & & \\
\hline different & $.20 \pm .41$ & $.21 \pm .43$ & .092 & .928 & .024 & .03 \\
\hline$t$ & 1.871 & 1.883 & & & & \\
\hline \multicolumn{7}{|l|}{ TIS-dynamic (10) } \\
\hline pre & $5.60 \pm 1.99$ & $6.00 \pm .96$ & .680 & .502 & & \\
\hline post & $6.87 \pm .83$ & $6.29 \pm .91$ & & & & \\
\hline different & $1.27 \pm 1.49$ & $.29 \pm .47$ & 2.360 & $.026^{*}$ & .81 & .60 \\
\hline$t$ & $3.300^{*}$ & $2.280^{*}$ & & & & \\
\hline \multicolumn{7}{|c|}{ TIS-coordination (6) } \\
\hline pre & $2.60 \pm .83$ & $2.64 \pm .84$ & .138 & .891 & & \\
\hline post & $3.40 \pm .63$ & $2.86 \pm .77$ & & & & \\
\hline different & $.80 \pm .67$ & $.21 \pm .43$ & 2.768 & $.01^{*}$ & .93 & .72 \\
\hline$t$ & $4.583^{*}$ & 1.883 & & & & \\
\hline \multicolumn{7}{|l|}{ TIS-total (23) } \\
\hline pre & $14.47 \pm 2.26$ & $14.93 \pm 1.33$ & .664 & .512 & & \\
\hline post & $16.73 \pm .96$ & $15.64 \pm 1.28$ & & & & \\
\hline different & $2.27 \pm 1.75$ & $.71 \pm .73$ & 3.076 & $.005^{*}$ & 1.00 & .79 \\
\hline$t$ & $5.013^{*}$ & $3.680^{*}$ & & & & \\
\hline
\end{tabular}

$* p<.05$,

STE group: selective trunk exercise group, TIS; trunk impairment scale

of abnormal compensatory strategies despite the functional restoration (Verheyden et al., 2009). In this study, the total effect size of the STE (Cohen $d$ ) shows TIS-coordination $d=.93$, TIS-dynamic balance $d=.81$, TIS-static balance $d=.02$, TUG $d=.79$, BBS $d=.73$. The average effect size of the study is $d=.66$ which lies between medium and large effect size (Cohen, 1994).

The TUG change of the intervention group in this study saw more improvement with 4.1 seconds than the control group at 2.01 seconds. The previous study (Kong et al., 2015), the patents with chronic stroke received balance training on the unstable surface show significantly increased in TUG from 18.32 seconds to 13.24 seconds.

In practice, the Minimal Detectable Change (MDC), decide the minimal effect of treatment (Liaw et al., 2008). MDC can be acceptable for $10 \%$ of the highest score gained
(Lu et al., 2008). The effect size of treatment (Cohen $d$ ) and MDC level were core indexes reflecting functional prognoses of the therapists and patients in clinical settings (Liaw et al., 2008; Lu et al., 2008).

The MDC of TUG in chronic patients with stroke was known to be between 2.9 and 5.2 seconds (Flansbjer et al., 2005). The highest record among the participants of the study was 10 seconds, compared to the average MDC, 1 second (less than 10\%), improving in the actual changes of 4.1 seconds. The change of BBS in the STE group scored 3.07 with an improvement of 3.1 percent. This was 1.71 (out of 56) higher than the control group's score of 1.36. The MDC of BBS was known to be lying between 2.5 and 4.13 (Liston and Brouwer, 1996; Flansbjer et al., 2012). The highest score of BBS in this study was 43 points by showing 4.3 of the effectiveness on average MDC 
and 3.07 of the actual changes. The STE probably enabled the paralyzed side to bear weight and to move in hemiplegic side by strengthening trunk flexion and extension, lateral flexion, rotation as well as controlling the proprioceptive sense. The selective movement of trunk and limbs had impacts on the center of gravity and movement and rotation motions which were required for sitting and standing in TUG and BBS. The STE activated trunk muscles as a proceeding controller and stabilized the trunk.

In the kinematic analysis of stroke patients, unstable pelvic movements and asymmetric patterns can be seen (Tyson, 1999). The physical therapists in rehabilitation have organized the trunk as the central key point of the body. The NDT principle control over the movement from proximal to distal body segments. Proximal stability of the trunk is a prerequisite for distal limb movement (Davis, 2003; Ryerson and Levit, 1997). Therefore, the improvement of proximal trunk control influences the functional activities such as standing and stepping (Davis, 2003; Verheyden et al., 2004; Verheyden et al., 2009).

The STE reinforce the symmetric pelvic movement and enable body weight to shift on the hemiplegic leg during walking. The TIS dynamic in STE group improved .98 compared to the control group. Verheyden et al. (2009) ascertained that 10 hours of additional exercise for trunk lateral flexion apart from routine treatment among sub-acute stroke patients improved 2.22 in TIC dynamic category; Karthikbabu et al. (2011a) discovered that the trunk exercise using a physio-ball was better than using a plinth with the improvement of 1.47 in TIS dynamic category among acute stroke patients.

The rotation of the trunk is rotations of the upper and lower parts of the trunk in the opposite directions of each other, measured by TIS-coordination category. In particular, the coordinated rotation of the lower trunk should shift body weight to the paralyzed side (Ryerson and Levit, 1997; Davis, 2003), TIS-coordination was improved with a .59 score (out of $6,9.8 \%$ ) in this study. Coordination of the trunk is stability task which requires counter rotation between the upper and lower trunk. The improvement of weight shift ability is important for coordination of the trunk (Davis, 2003; Messier et al., 2004). The rotation of the lower part of the trunk is more difficult for patients with stroke (Davis, 2003; Verheyden et al., 2009).

Karthikbabu et al. (2011a) reported that the trunk exercise using a physio-ball was improved with a 1.33 score in the TIS dynamic category, compared to using a plinth among acute stroke patients. However, the TIS-static category, which was easily conducted in comparison with TIS-dynamic and coordination categories for the participants, did not show a significant difference between the effect size of pre-test and post-test, having 6 out of 7 points.

According to a posturographic analysis from Messier et al. (2004), compensatory movements using the upper trunk were frequently seen, while the movement of the lower trunk (pelvis) was rarely reported. In a practical view, the lower trunk rotation is very difficult amongst stroke patients (Verheyden et al., 2005). It is suggested that this particularly difficult movement in stroke is possibly caused by a combination of factors such as proximal hypertonia in the lower extremities or paresis of the trunk muscles and anteroposterior pelvic obliquity and rotation (Davis, 2003; Messier et al., 2004). However, the patients had some troubles when they shifted the center of gravity from sitting or standing to their paralyzed side (Van Nes et al., 2008; Chern et al., 2010). There may be a possibility exists that STE training may elevate symmetrical pelvic movement, thus better weight shifting towards hemiplegic limb during gait.

Mudie et al. (2002) ascertained that stroke patients in the early stages could see improvement in symmetric sitting position by using recognition training of the trunk location in their study. Therefore, lateral flexion and rotation exercise for controlling the trunk, which were applied in the study, were effective since the paralyzed side was able to tolerate bearing the body weight. Moreover, the effect 
sizes of trunk training were higher than usual physiotherapy, having $d=.81 \sim .93$ in TIS-dynamic and coordination respectively. However, there is strong evidence in the stroke literature that trunk performance is an important predictor of functional outcomes (Verheyden et al., 2004; Verheyden et al., 2009). In this study, the effectiveness of the trunk exercise was detected by the improvement in mobility, balance, and trunk control in comparison to the control group. In a neurological development view, the trunk is the center of a body, and the movements of the four limbs precede from proximal to distal parts (Davis, 2003). The trunk plays an important role in the body as a central axis for functional movements of the upper and lower limbs and stabilizing proximal movements in order to perform distal movements properly. Through this process, if controlling proximal trunk is enhanced, there will be improvements in the preceding posture control for mobility and balance as well as the movements of the four limbs. As a result, the trunk exercise could have more intensive effects of treatment than the general training since it consists of task-based training specialized by the individuals' needs. The limitation of the study is that the study power could not reach the statistical significance, the standard known as between average .48 and .8 , because of the small sample size. Thus, it is difficult to generalize the results of this study about the effectiveness of the trunk exercise due to the small number of participants and the short period of the study. For future studies, it is important to verify the effect of the trunk training with the surface electromyography in order to measure selective activation of trunk muscles by applying it to a larger number of patients.

The limitations of this study were a short period of exercise, a small sample size $(n=15)$ and it consisted only of patients diagnosed with the disease for 13 months. The study ascertained that the functional mobility, balance, and trunk control ability were improved by the effect of STE.

\section{Conclusions}

The disorders among stroke patients cause negative impacts on performing functional movements of the body. The result of the study shows that the total effect size of the trunk exercise is Cohen $d=.66$ and is effective on TIS-coordination $(d=.93)$, TIS-dynamic $(d=.81)$, TUG $(d=.75)$ and BBS $(d=.73)$. The training of trunk control accompanied with functional exercises have positive effects on separate movements of the upper and lower trunk and lateral rotation, coordinated movements of limbs. Further future studies should be conducted with randomization and a larger sample in order to investigate the effectiveness of trunk exercise by using surface electromyography.

\section{Reference}

Bohannon RW. Lateral trunk flexion strength: impairment, measurement reliability and implications following unilateral brain lesion. International Journal of Rehabilitation research. 1992;15(3):249-51.

Chern JS, Lo CY, Wu CY, et al. Dynamic postural control during trunk bending and reaching in healthy adults and stroke patients. Am J Phys Med Rehabil. 2010; 89(3):186-97.

Cohen J. "The earth is round $(\mathrm{p}<.05)$ " American Psychologist. 1994;49(12):997-1003.

Davis PM. Problems associated with the loss of selective trunk activity in hemiplegia. Right in the Middle. 2003: 31-66.

Dickstein R, Heffes Y, Laufer Y, et al. Activation of selected trunk muscle during symmetric functional activities in post stroke hemiparetic and hemiplegic patients. Journal of Neurology, Nurosurgery, and Psychiatry. 1999;66(2):218-21.

Dickstein R, Sheffi S, Ben Haim Z, et al. Activation of flexor 
and extensor trunk muscles in hemiparesis. Am J Phys Med Rehabil. 2000;79(3):228-34.

Duarte E, Marco E, Muniesa JM, et al. Trunk control test as a functional predictor in stroke patients. J Rehabil Med. 2002;34(6):267-72.

Flansbjer UB, Blom J, Brogardh C. The reproducibility of Berg Balance Scale and the Single-leg Stance in chronic stroke and the relationship between the two tests. PM R. 2012;4(3):165-70.

Flansbjer UB, Holmback AM, Downham D, et al. Reliability of gait performance tests in men and women with hemiparesis after stroke. J Rehabil Med. 2005;37(2): 75-82.

Fujiwara T, Sonoda S, Okajima Y, et al. The relationships between trunk function and the findings of transcranial magnetic stimulation among patients with stroke. J Rehabil Med. 2001;33(6):249-55.

Han KB, Shin WS. Effects of Trunk Position Sense through Visual Cue Deprivation Balance Training in Subacute Stroke. J Korean Soc Phys Med. 2013;8(3):327-35.

Hopkins WG. Measures of reliability in sports medicine and science. Sports Med. 2000;30(1):1-15.

Jang JY, Kim SY. Effects of trunk control exercise performed on an unstable surface on dynamic balance in chronic stroke patients. J Korean Soc Phys Med. 2016;11(1): $1-9$.

Karatas M, Cetin N, Bayramoglu M, et al. Trunk muscle strength in relation to balance and functional disability in unihemispheric stroke patients. Am J Phys Med Rehabil. 2004;83(2):81-7.

Karthikbabu S, Nayak A, Vijayakumar, K, et al. Comparison of physio ball and plinth trunk exercises regimens on trunk control and functional balance in patients with acute stroke: a pilot randomized controlled trial. Clin Rehabil. 2011a;25(8):709-19.

Karthikbabu S, Rao BK, Manikandan N, et al. Role of Trunk Rehabilitation on Trunk Control, Balance and Gait in Patients with Chronic Stroke: A Pre-Post Design.
Neuroscience \& Medicine. 2011b;2:61-7.

Kong HN, Bang DH, Shin WS. Effects of Balance Training on Different Support Surface on Balance and Gait in Patients with Chronic Stroke. J Korean Soc Phys Med. 2015;10(3):275-83.

Liaw LJ, Hsieh CL, Lo SK, et al. The relative and absolute reliability of two balance performance measures in chronic stroke patients. Disabil Rehabil. 2008;30(9): 656-61.

Liston RA, Brouwer BJ. Reliability and validity of measures obtained from stroke patients using the Balance Master. Arch Phys Med Rehabil. 1996;77(5):425-30.

Lu WS, Wang $\mathrm{CH}$, Lin $\mathrm{JH}$, et al. The minimal detectable change of the simplified stroke rehabilitation assessment of movement measure. J Rehabil Med. 2008;40(8):615-9.

Mao HF, Hsueh IP, Tang PF, et al. Analysis and comparison of the psychometric properties of three balance measures for stroke patients. Stroke. 2002;33(4): 1022-7.

Messier S, Bourbonnais D, Desrosiers J, et al. Dynamic analysis of trunk flexion after stroke. Arch Phys Med Rehabil. 2004;85(10):1619-24.

Mudie MH, Winzeler-Mercay U, Radwan S, et al. Training symmetry of weight distribution after stroke: a randomized controlled pilot study comparing task-related reach, Bobath and feedback training approaches. Clin Rehabil. 2002;16(6):582-92.

Podsiadlo D, Richardson S. The timed "Up \& Go": a test of basic functional mobility for frail elderly persons. J Am Geriatr Soc. 1991;39(2):142-8.

Ryerson S, Byl NN, Brown DA, et al. Altered trunk position sense and its relation to balance functions in people post-stroke. J Neurol Phys Ther. 2008;32(1):14-20.

Ryerson S, Levit K. Functional movement: A practical model for treatment. Functional movement reeducation. Churchill Livingstone. 1997:1-14.

Song JM, Kim SM. The effect of trunk stability exercise on 
balance and gait in stroke patients. J Korean Soc Phys Med. 2010;5(3):413-20.

Tsuji T, Liu M, Hase K, et al. Trunk muscles in persons with hemiparetic stroke evaluated with computed tomography. J Rehabil Med. 2003;35(4):184-8.

Tyson SF. Trunk kinematics in hemiplegic gait and the effect of walking aids. Clin Rehabil. 1999;13(4):295-300.

Van Nes IJ, Nienhuis B, Latour H, et al. Posturographic assessment of sitting balance recovery in the subacute phase of stroke. Gait Posture. 2008;28(3):507-12.

Verheyden G, Nieuwboer A, Feys H, et al. Discriminant ability of the Trunk Impairment Scale: A comparison between stroke patients and healthy individuals. Disabil
Rehabil. 2005;27(17):1023-8.

Verheyden G, Nieuwboer A, Mertin J, et al. The Trunk Impairment Scale: a new tool to measure motor impairment of the trunk after stroke. Clin Rehabil. 2004;18(3):326-34.

Verheyden G, Vereeck L, Truijen S, et al. Trunk performance after stroke and the relationship with balance, gait and functional ability. Clin Rehabil. 2006;20(5): 451-8.

Verheyden G, Vereeck L, Truijen S, et al. Additional exercises improve trunk performance after stroke: a pilot randomized controlled trial. Neurorehabil Neural Repair. 2009;23(3):281-6. 
